\title{
16 \\ TASK ORIENTATION IN USER INTERFACE DESIGN
}

\author{
Michael Gritzman*, Anders Kluge* and Hilde Lovett* \\ Norwegian Computing Center \\ Gaustadallèen 23, P.O.Box 114 Blindern \\ N-0314 Oslo, Norway \\ *E-mail:\{Michael.Gritzman, Anders.Kluge, Hilde.Lovett\}@nr.no
}

KEY WORDS: User interface, task orientation, consumer electronics, dialogue design, design principles, object-action, smart phone.

\begin{abstract}
This article outlines some ideas for the design of user interfaces for computer systems and consumer electronics used for specific and well-defined tasks. The basis of the discussion is found in Norman's notion of task orientation. Prevailing design approaches, namely standardisation efforts and rules for human-computer interaction, are criticised. The alternative presented is specialisation of interaction mechanisms in accordance with the task, and user guidance with a limited set of possible actions. The article is mainly theoretical, but includes a concrete design of two prototypes for different communication services as examples.
\end{abstract}

\section{INTRODUCTION}

Rules for dialogue design as formulated by Shneiderman (Shneiderman, 1987) and Nielsen (Nielsen, 1990) have been widely accepted by the researchers in the Human-Computer Interaction field. These rules together with the various standardisation efforts within the user interface area (Apple, 1987; IBM, 1988; Open Software Foundation, 1991) have had a profound impact on the development of systems and user interfaces during the last ten years. Applications and window-systems based on these standards and rules for interface design have been highly successful in bringing new users to the computer and in extending use for the people already using computers. However, when the chip enters the arena of consumer electronics and the computer is used for such different tasks as sending a fax, sending and receiving mail, paying bills, watching video or listening to a Shakespeare play, it is not standardisation and rigid rules for interaction which give the users the best opportunities to utilise their computers or other electronic devices. The extended use of the computer together with the computerisation of consumer devices create new demands for the development of user interfaces (Brouwer-Janse, 1992; Gritzman et al., 1994).

The present paper deals with computer systems and consumer electronics which are used for one or a limited set of specific and well-defined tasks. Standard systems such as word-processing, spreadsheets and accounting systems are not taken into account in the argument. In these standard systems the user is immersed in the use of the systems for a longer period of time, and the system has a complex set of commands and is used for a wide range of tasks.

In the INNITEL project, two prototypes were developed both offering the same range of services to the public: traditional telephone and directory services, home banking, and voice mail. One of the prototypes simulates these services on a "smartphone"; the second prototype is meant for use in a Windows environment on a regular PC. The starting point of the project was acknowledging the necessity of engaging in approaches alternative to traditional user interface design, when designing the user interface for these kinds of services. In fact, the design process was not guided by any style guide or user interface standard, and even widely accepted rules for good user interface design (Nielsen, 1990; Shneiderman, 1987) were in some cases violated.

The notion of task orientation, as suggested by Norman (Norman, 1988), is proposed as the basis of a different approach. In the task-oriented setting only the user and the task at hand guide the design of the user interface. From the user's perspective the nature of the screen changes from the virtually unrestricted 'object-action' - choose an object and then choose what to do with that object - which leaves the user as the initiator of all actions to be taken throughout the 
dialogue, to the what-you-see-is-what-you-can-doright-now perspective, where the user is led through the task. Actions which are not crucial in a given situation in order to accomplish a certain task or subtask are not visible for the user. Some new design principles, dialogue mechanisms and ideas within the framework of task orientation are suggested based on the actual design of the INNITEL prototypes. There are at this point no empirical data from user testing of these prototypes.

\section{NORMAN'S NOTION OF TASK- ORIENTATION}

Donald Norman has often stressed his position on or rather opposition to - the thinking behind the design of computer applications, including state-ofthe-art graphical user interfaces. He even opposes the emphasis on design of user interfaces, since he considers the user interface to be a kind of obstacle the user has to overcome in order to accomplish her goal. He wants the task and the tools being used to accomplish that task to be visible to the user (Norman, 1990).

The basis of this position is that virtually all modern applications with their graphical user interface which often adheres to certain user interface standards - are not directed towards the user and her task. The user is forced to focus on the user interface, not the task. It seems that in this view the user interfaces reflect the computer itself and the structure of the systems rather than the tasks to be accomplished. The user must concentrate on how to manipulate icons and spends time and effort manoeuvring in complex structures such as tree structures. She enters the world of technology in adapting to concepts such as file and file system, window and window system, menu and menubar, etc.

Norman suggests task-orientation as the way we ought to think when we design software applications to be used by people, a process he correctly denotes product design: "Product design should start off by getting the priorities right: 1. The user - what does the person really need to have accomplished? 2. The task - analyse the task. How best can the job be done?, taking into account the whole setting in which it is embedded ... 3. As much as possible, make the task dominate; make the tools invisible. 4. Then get the interaction right ..." (Norman, 1990).

Norman refers to games on specialised computer game machines as an example of task-oriented design: "Why is Nintendo, the game machine, so superior to the more powerful and flexible computer? Nintendo is also a computer, simply disguised as a game. The users just want to play a game, and Nintendo lets them do that simply, directly." Norman recognises of course that the key factor in its success is its specialisation, and that the range of use is very limited, but this must be a trade-off: "Generality and power versus specialisation and ease of use" (Norman, 1990).

\section{FROM USER INITIATED INTERACTION TO "WHAT-YOU-SEE-IS-WHAT-YOU-CAN-DO- RIGHT-NOW"}

There are two fundamental paradigms structuring the human-computer interaction on the Apple Desktop Interface, according to Apple's own presentation of its concept. The first paradigm is that users first select an object (often in the form of icons or other graphical representation on the screen), then they select an action to be taken on the chosen object (in the menus). The second paradigm is that users can select an object, then drag it into another object which has an action associated with it (Apple, 1987). The famous wastebasket is an example of an object with an associated action. The idea of first selecting an object, then choosing an action to be taken on it, has been copied into virtually all other major graphical user interface standards. In the user interface standard issued by IBM the principle was called object-action (IBM, 1988), a designation adopted in the present discussion. Both paradigms reflect the same idea of the user choosing what to do among the variety of feasible combinations of objects and actions. The philosophy behind the object-action paradigms is to put the user in control. At any time the user is free to choose which action to take on the object of her choice. Of course, the systems prevent the user from performing illegal combinations of objects and actions, but in principle the user is at any given time the initiator. Apple makes it explicitly a general design principle: "The user, not the computer, initiates and controls all actions" (Apple, 1987).

It is beyond the scope of the present discussion to evaluate the suitability of this design principle in general, presumably it is a desired quality for general purpose computers. However, the basic assumption of putting the user in the position of the initiator and controller of all action must be questioned when the sole goal of a person is to perform a very specific task, such as paying a bill, calculating a sum or sending a fax. In such a setting it may prove counterproductive to make the user the initiator of actions to be taken. An example of the latter is the airport check-in public access terminal. The user approaches the terminal with no other purpose than to be checked in effectively and quickly on her 
flight. She might not have prior experience in performing that task on this machine. She does not want to initiate action; she expects the system to lead her through the task (Gritzman et al., 1993). This argument does not reject object-action as a useful instrument in the user dialogue on standard systems such as word-processing, accounting systems, etc. What is questioned is the suitability of this concept when used for limited, well-defined tasks or on other consumer devices such as the smart-phone.

In the arguments presented by Apple in support of the user control and initiative principle, situations where this is not the case are described: "Too often, however, the computer acts and the user merely reacts within a limited set of options. In other instances, the computer 'takes care' of the user, offering only those alternatives that are judged 'good' for the user or that protect the user from detailed liberations" (Apple, 1987). The description here applies very well to the task-orientated design approach. The user who is only concerned about her task wants to be taken care of; she wants to be offered only "good" alternatives and she wants to be protected from detailed liberations. Consequently, she should not be offered an array of possible actions at any given time, only the necessary means to get on with the well-defined task. Such an environment of use will be denoted "what-you-see-is-what-youcan-do-right-now", the screen only offering actions appropriate in relation to the actual task. It is the task which defines the sequence of actions to be taken. In the above example of the airport check-in terminal the traveller is led through a well defined sequence of actions: first she chooses the language for the dialogue, then places her ticket in the optical reader, then confirms the flight when it is identified by the machine as correct, then chooses a seat, etc. The system initiates the action; the user is being taken care of by the computer.

The conclusion seems inescapable that another overall perspective is needed when our design efforts shift focus from general purpose computer systems to computer systems for dedicated tasks or to other computerised devices. The functionality-focused object-action paradigms must be changed to a taskfocused perspective. In the following paragraph some elements of the user interface of the INNITEL prototypes are presented. These prototypes were designed with what-you-see-is-what-you-can-doright-now as the overall design guideline. No attempt is made to describe the prototypes in their entirety; instead, elements of the design, including innovative dialogue mechanisms, are presented.

\section{THE TASK-ORIENTED DESIGN OF THE INNITEL PROTOTYPES}

Two prototypes were developed, both on Windowsbased PC's, within the INNITEL project: a version for smart-phone and a version to be used on a regular $\mathrm{PC}$ with Windows. Both prototypes were designed for home use or occasional use in small businesses. The smart-phone imagined here is a telephone with a small graphical, high colour resolution, touchsensitive screen and a small pull-out keyboard. The prototypes simulate the same services: (1) extended telephone services, (2) directory services, (3) home banking and (4) voice mail. Extended telephone services comprise services such as early morning calls, call forwarding, etc. Electronic directory services are the electronic version of searching for numbers in a paper-based telephone directory. Home banking provides the consumer with the possibility of paying bills and obtaining personal account information. Voice mail is a system for sending and receiving audio messages.

The smart-phone version was developed first; the design principles and ideas which had emerged during the design process were subsequently transferred to the PC-version. From the very beginning it was decided that the design process should not be directed by any user interface standard or even generally accepted guidelines for user interface design. On the contrary, guidelines, design concepts and dialogue mechanisms were developed specifically for this application during the construction of the prototypes. This way of designing user interface was inspired by Nelson's notion of "Design of principles" (Nelson, 1990). The objectives were (1) to design principles of interaction in accordance with the purpose of the system (the task from the user's point of view), and (2) to design principles to make the prototypes appear as a clear and unambiguous whole for the user. The guidelines, concepts, and mechanisms are described and discussed in the following. Finally, a list of well-known design concepts and dialogue mechanisms which were omitted in the INNITEL prototypes, is presented. It is argued that they are not appropriate means of interaction in the taskoriented environment.

\section{Touch On}

A central design principle which emerged during the development of the smart-phone version is denoted touch-on, referring to the main device of interaction on this medium: touch directly on the touchsensitive screen. The meaning of touch-on is twofold: (1) To touch on virtually any part of the screen will cause an appropriate system event, or (2) to go 
on by touching on, which means to touch on a relevant place on the screen to instigate the next step. The first meaning of this design principle indicates that the whole screen must be prepared to respond when the user touches any part of it; hence there must be a direct and intuitive coherence between the point on the screen which is touched on and the system action triggered by the "touch". The second meaning implies that touching on the screen indicates what the user wants to do next. Thus this design principle constitutes an implicit sequencing mechanism. Actually, touch on is the only sequencing mechanism in the smart-phone version of the INNITEL prototypes.

In the PC-version the same idea applies, with a slightly different approach due to the difference between touch screen and mouse control. A click at any place on the screen must still cause an intuitive coherent system action, and the only sequencing mechanism is still to click on what you want to do next. The difference is that the mouse functions in two phases. First the cursor is moved to a certain point on the screen, which may trigger a system action, and subsequently the user can click on that spot, which may trigger another system action.

The INNITEL prototypes have no undo-function. In the task-oriented environment the user constantly takes well-defined actions directed towards the fulfilment of the task at hand. To undo is redefined as to $d o$ something else, to take action which in practise would undo a former action taken. An example from the home banking services offered in INNITEL is if the consumer has mistakenly paid a bill, which in fact means that a message is sent to the bank to pay the bill on a certain date. The consumer must now actively enter her list of bills waiting to be paid by the bank and remove the bill from the list. She must touch on - in both meanings of the principle - in order to reverse the former actions taken.

\section{Design Concepts and Dialogue Mechanisms}

Some main design concepts and dialogue mechanisms used in the INNITEL prototypes are described below. The list is far from complete. Rather, the examples illustrate typical concepts and some innovative ideas in those particular taskoriented user interfaces. A common aspect of the elements on the list is their goal of preserving the environment in which a service is offered to the user: the user remains within the same context of use while accomplishing a task.
Fixed screen-structure The structure of the screen is always the same regardless of what service is chosen and which stage the user has reached in the service. Accordingly, the contents of the screen change in connection with the service chosen and the stage reached in the service. In the smart-phone version the screen is divided into three areas: the input area, the information area and the action area. A special background colour is associated with each area and the size of the areas are constant, independent of the functionality of the different services. The three-part division of the screen is also applied to the PC-version, the same functions being associated with the same areas.

Receipts In the smart-phone version of the INNITEL prototypes, screen elements representing a task or a sub-task are stamped like a receipt when they are accomplished. If the task is to order an early morning call, the screen element showing the clock set by the user - is stamped "ordered" to confirm that the call is ordered. When paying a bill, the screen element showing the bill is stamped "sent to payment" (the bill is actually paid on a particular date determined by the payer when ordering the bill to be paid). The decisive difference between the receipt stamped directly on a screen-element and the conventional dialogue box is that the latter is a feature external to the task. The user's attention is distracted in order to deal with this new screen element. She is forced to relate to the contents of the dialogue box i.e. by reading a message or answering a question. Subsequently she must take action to remove it from the screen. In the case of the receipt, the user still touches on the place at the screen representing what she wants to do next, and is not distracted from the context of use. In the PC-version receipts are used in a slightly different manner: a special area on the screen is dedicated for messages which communicate to the user that the task - or subtask - at hand is accomplished.

Information signals Even in a task-oriented environment, there might occasionally be a need to communicate information not directly connected to the task at hand to the user. In the INNITEL prototypes this is the case when information concerning other services with a direct impact on the user must be communicated at once. The user wants to be notified immediately about a new incoming audio-message in her voice-mail box, and also wants easy access to information about the state of the telephone, i.e. if calls are forwarded to another number. The information signals are a means to communicate such information which is external to the task without interrupting the present task and 
without distracting the user. The information signals are an integrated and fixed part of the screen, and are incorporated into the context of use. In the smartphone version they have the form of a circle placed in the action area divided into a number of sections, each of which becomes yellow when that particular area holds information. In the PC-version, the information signals are an array of small circles in a designated area on the screen, turning yellow as in the smart-phone version when they hold information.

The pop-up information box This dialogue mechanism is only used in the touch-screen environment of the smart-phone version. An information box, which may cover the whole screen, pops up while the user touches the graphical element on the screen associated with it. In this case the yellow circle described above is the associated screen-element. If the information signals (here the yellow circle) indicate a new incoming audiomessage, the pop-up information box may hold information about the audio-message received such as the sender, the time it was received, etc. The motivation for using this kind of dialogue mechanism is to limit the user's distraction from the context of use, which is the task at hand. By using this dialogue mechanism the need for manoeuvring between different screen-pages is removed. The user is automatically returned to the previous situation, when she removes her finger. In the PC-environment the pop-up information box is not used. Instead, information is shown in specific areas on the screen, while the cursor is placed over the corresponding area on the screen.

Design Concepts and Dialogue Mechanisms omitted in INNITEL

In the same manner as in the case above there has been made no attempt to construct a total list of design concepts or dialogue mechanisms which were purposely omitted in the INNITEL prototypes. Rather, the examples in the list are meant to illustrate type of concepts and mechanisms which cannot be included in a task-oriented environment. Among the reasons for abandoning these concepts and dialogue mechanisms are keeping the user interface as simple as possible, preserving the context of use and avoiding any element on the screen which, from the user's - or consumer's - point of view, is not directly intended to help her to accomplish her task.

No explicit help function In the INNITEL prototypes no explicit help is offered. At every step in the dialogue the screen in its entirety offers a maximum of help. This is possible because the screen always focuses on the next specific action, well defined within the task, to be taken by the user. Situations where the context of use changes from the chosen task to the help system, are eliminated.

No Menus INNITEL offers no menus, i.e. the prototypes make no explicit use of this concept, thereby eliminating features like menubar or menu hierarchy from the user interface. The basic idea behind the introduction of the concept menu to computer systems is to stress the fact that users can choose within a wide spectrum of functionality, with systems rich in features. Within the task-focused what-you-see-is-what-you-can-do-right-now perspective the consumer is not offered a variety of options within the system; on the contrary, features not directly connected to the specific state of performing the task are purposely hidden. In hierarchical menu systems the user manoeuvres through levels of menus in order to reach a place in the system where a certain action may be taken. In the task-oriented environment manoeuvring activities should be avoided.

No dialogue boxes Dialogue boxes - a concept well-known from various graphical user interface standards - are not used in the INNITEL prototypes. The main objection to dialogue boxes in the taskoriented user environment is that they constitute an artificial layer between the user and her task. The user must often take specific action to remove the dialogue box when it is no longer needed. Such an action is not task-driven; the dialogue box becomes a new context of use which the user must react to.

No set of Screenpages A typical example of the machine-oriented thinking of many applications is the concept of the system as a set of screenpages. The users must expend a great deal of energy manoeuvring between screenpages in the system, which is unnecessary for the accomplishment of a task. In the INNITEL prototypes the concept of screenpage does not exist; the design aims at getting the user to comprehend the screen as the arena where certain services are offered. Since there are no screenpages in INNITEL there is no need for explicit sequencing mechanisms. Explicit sequencing mechanisms are defined here as specific means by which the user moves around between screen-pages such as buttons labelled "next", "back", etc.

\section{CONCLUSIONS}

Computer use is spreading in the population in two important ways: by development of different kinds of personal computers and by the computerisation of consumer devices. Computers will be everywhere and everything will have a computer inside. An 
important consequence of this development is that people will use computers in a wide range of different contexts and environments. Another consequence is that the spectrum of types of applications will be immense. This development, often denoted ubiquitous computing, challenges the prevailing opinion of user interface design.

The concept of task orientation provides two primary solutions in the sphere of human-computer interface design: (1) specialisation, meaning interaction mechanisms and devices that are suited for fulfilling one particular task, and (2) guidance, meaning that the user interface should at any given time be directed towards giving the user maximum help in choosing among a limited set of relevant possibilities to fulfil the task.

Specialisation is in many ways the opposite of standardisation. A toaster and a TV-set are standardised on the technical level with regard to voltage and plugs, but differ in interaction devices and mechanisms. Standardisation has its place on the technical level, but not in user interface design. The two different tasks confronting a user when applying the devices have resulted in different interaction mechanisms. Similarly, the interaction mechanisms the user needs in order to send a fax and to listen to a play also generate different requirements for interaction mechanisms. The challenge is to derive a set of principles which may be unique for a task, to guide the design towards usable and useful applications and devices.

The concept of guidance includes both the what-yousee-is-what-you-can-do-right-now principle and the touch on-principle described above. User guidance is especially relevant to the use situation. A multitude of possibilities is not what a user needs when confronted with a task in a complex use setting with many other phenomena requiring attention. "People aren't trying to use computers they're trying to get their jobs done" (Apple, 1987).

\section{ACKNOWLEDGEMENTS}

The INNITEL project was funded by Norwegian Telecom Research. We are indebted to Trond Bechmann for his encouragement throughout the project. Dag Belsnes, Ivar Solheim and Shari Nilsen provided valuable comments on the manuscript.

\section{REFERENCES}

Apple, (1987). Human Interface Guidelines: The Apple Desktop Interface, AddisonWesley Publishing Company.
Brouwer-Janse, M.D. (moderator), (1992). Interfaces for consumer products: "How to camouflage the computer?", in Penny Bauersfeld, John Bennett, and Gene Lynch (eds.) Human Factors In Computing Systems, Striking a Balance, CHI'92 Conference Proceedings, acm Press.

Gritzman, M., Kluge, A., Lindsjørn, Y., Lovett, H., Storrøsten, M., (1993). Vennlige publikumsterminaler, NR-rapport no. 865, Norwegian Computing Center (in Norwegian).

Gritzman, M., Kluge, A., Lovett, H., (1994). Design Principles for the Human-Computer Interaction on a screen-Based Telephone, in Kresten Berg \& Kim Borreby (eds.) HomeOriented Informatics, Telematics \& Automation, Proceedings, University of Copenhagen

IBM, (1988). Systems Application Architecture, Common User Access, Panel Design and User Interaction, IBM Corporation.

Nelson, T.H., (1990). The Right Way to Think About Software Design, in Brenda Laurel (ed.) The Art of Human-Computer Interface Design, Addison-Wesley Publishing Company, pp. 235-244.

Nielsen, J., (1990). Traditional Dialog Design applied to Modern User Interfaces, Communications of the ACM/October 1990/Vol. 33, No. 10, pp. 109-118.

Norman, D.A., (1988). The Psychology of Everyday Things, New York: Basic Books.

Norman, D.A., (1990). Why Interfaces Don't Work, in Brenda Laurel (ed.) The Art of Human-Computer Interface Design, Addison-Wesley Publishing Company, pp. 209-219.

Open Software Foundation, (1991). OSF/Motif, Style Guide, Prentice Hall, Englewoods Cliffs, New Jersey.

Shneiderman, B., (1987). Designing the User Interface, Strategies for Effective HumanComputer Interaction, Addison-Wesley Publishing Company. 